\title{
Percepción social y respuesta institucional frente al desastre de Tierradentro, Colombia
}

\author{
Social Perception and Institutional Responses to the Colombian \\ Tierradentro Region Disaster
}

\section{Laura Astrid Ramírez Elizalde ${ }^{1}$ Irene Vélez Torres ${ }^{2}$}

Para citar este artículo, utilice el nombre complete así:

Ramírez Elizalde, L. A. \& Vélez-Torres, I. (2015). Percepción social y respuesta institucional frente al desastre de Tierradentro, Colombia. Perspectiva Geográfica 20(2),

\section{Resumen}

En junio de 1994, un sismo sacudió el suroccidente de Colombia, el cual, sumado a las fuertes lluvias y a las condiciones geofísicas de la zona de Tierradentro, desencadenó una serie de avalanchas, generando un evento complejo que dejó más de mil muertos y una severa destrucción de viviendas y cultivos, cuyos afectados fueron, en su mayoría, indígenas nasa. Este artículo analiza, bajo un enfoque histórico y territorial, cómo se gestó la respuesta institucional frente al desastre de Tierradentro, y cómo esta respuesta se articuló con la percepción social del desastre que tuvo la comunidad afectada y los medios de comunicación. Para responder a esta pregunta se siguió una metodología que vinculó etnografía, cartografía social y revisión de archivo.

Palabras clave: Desastre de Tierradentro, percepción social del riesgo, respuesta institucional frente al desastre, Tierradentro.

1 MsA en Geografía, Universidad de los Andes. Investigadora independiente. lauraastrid.ramirez@gmail.com, la.ramirez87@ uniandes.edu.co

2 Profesora EIDENAR, Facultad de Ingeniería, Universidad del Valle. PhD en Geografía Política, University of Copenhagen. Docente e investigadora del EIDENAR, Facultad de Ingeniería, Universidad del Valle. irene.velez@correounivalle.edu.co 


\section{Abstract}

In June 1994, an earthquake hit the south-west of Colombia. In combination with a heavy rainfall and the particular geo-physical conditions of the Tierradentro area, a series of avalanches led to more than a thousand deaths and the severe destruction of homes and crops; where the Nasa indigenous people were the most severely affected. Using a historical and territorial approach, this paper inquires into the institutional response to the Tierradentro disaster, and how the social perception of the disaster was articulated amongst the affected community and media. To answer this challenge, a methodology was employed that linked ethnography, social mapping and archive revision.

Keywords: Colombia's Tierradentro Disaster, Social Risk Perception, Institutions Response. 


\section{Introducción}

El 6 de junio de 1994, el suroccidente colombiano fue impactado por un sismo de magnitud 6,4 en la escala de Richter; el epicentro, a 10 kilómetros de profundidad, se ubicó en el corregimiento de Dublín, municipio de Páez, departamento del Cauca; esta zona montañosa contiene una cuenca hidrográfica cuyo eje es el río Páez (ver Figura 1), su topografía es accidentada y se caracteriza por profundas depresiones, hondonadas, valles estrechos, pequeñas terrazas y altiplanicies (Pachón, 1996); al norte se impone el volcán nevado del Huila, cuyo pico central corresponde al punto más alto de los Andes colombianos $(5.750 \mathrm{msnm})^{3}$. La irregularidad del terreno, característica de esta zona de la cordillera, genera gran variedad de temperaturas e influye en la cantidad y magnitud de las lluvias. Así, las características de este espacio, las fuertes lluvias y el sismo desencadenaron una serie de deslizamientos de tierra que represaron los ríos y produjeron avalanchas que tuvieron gran impacto socio-ambiental (Olson y Sarmiento, 1995). Este evento complejo produjo lo que se ha conocido como el desastre de Tierradentro.

Este artículo indaga la espacialidad del riesgo y del desastre desde una

3 Datos tomados de la página oficial de Ingeominas: www.ingeominas.gov.co. perspectiva político- institucional histórica. Bajo un enfoque territorial (Agnew, 1994), se busca comprender cómo se gestó la respuesta institucional frente al desastre de Tierradentro y cómo esta respuesta se articuló con la percepción social del desastre que tuvo la comunidad afectada y los medios de comunicación. A partir de esta pregunta de investigación, se expone la relación que se estableció entre la respuesta gubernamental y las comunidades afectadas, la cual estuvo marcada por la creación, por la Presidencia de la República, de la Corporación Nasa Kiwe, el 9 de junio de 1994. Desde entonces, fue esta la institución la encargada de coordinar las acciones interinstitucionales tendientes a recuperar y rehabilitar de manera integral las condiciones sociales, económicas y culturales de los damnificados ${ }^{4}$.

La zona conocida como Tierradentro está conformada por los municipios de Páez e Inzá, ambos ubicados en el departamento del Cauca. En 1994, estos municipios tenían 30.679 y 20.624 habitantes, respectivamente, en su mayoría población indígena localizada en sectores rurales con vocación agrícola. La tragedia dejó como resultado cerca de 1.100 muertos, más de 40.000 ha de tierra destruida y alrededor de 7.000 per-

4 Ver la historia, misión y objetivos de la Corporación Nasa Kiwe en: http://www.nasakiwe.gov.co/. También en Wilches-Chaux, 1995 y 2000. 
sonas que fueron reubicadas 5 . Dado que el $80 \%$ de los afectados fueron indígenas nasa, el carácter étnico de esta población fue central en la respuesta al desastre, máxime si se tiene en cuenta el contexto histórico en el que recientemente se había construido una nueva carta constitucional (1991), que reconoció el país como pluriétnico y multicultural.

Según UNISDR y la Corporación OSSO (2013, p. 39), el caso de Tierradentro ocupa el octavo lugar como desastre con mayor pérdida de vidas humanas por manifestación intensiva del riesgo ${ }^{6}$ en América Latina entre 1990 y 2011. Este dato es relevante para comprender la magnitud de la tragedia y, en el mismo sentido, destacar su estudio como un caso representativo que, por su magnitud y por las características de la zona y de la población afectada, merece ser analizado. El presente artículo no tiene por objeto estudiar el complejo fenómeno geofísico desencadenado por el sismo, su propósito se centra en comprender la respuesta institucional y las percepciones sociales del

5 Ver: DNP, 2004; Gómez y Ruiz, 1997. Y sobre el proceso de reubicación de comunidades nasa en los resguardos de Mosoco y Musse Ukwe ver Ramírez, 2013.

6 Se llaman manifestaciones intensivas del riesgo a aquellas que impactan grandes concentraciones de población y actividades económicas, y que se caracterizan por generar gran cantidad de pérdidas de vidas humanas y bienes materiales. Los impactos generados por estas manifestaciones del riesgo pueden ser considerados catástrofes o desastres (UNISDR y la Corporación OSSO, 2013, p. 14) riesgo y del desastre que se tejieron alrededor del trágico evento. Este énfasis aporta a los estudios sobre prevención y atención de desastres, evidenciando de manera concreta los mecanismos de respuesta -gubernamentales, institucionales, sociales $y$ culturales- que se pusieron en marcha. El análisis abarca un marco temporal breve: los dos meses siguientes a la tragedia, en tanto que la construcción de mecanismos de respuesta institucional se concentra en este periodo coyuntural; no obstante, vale destacar que algunos de estos mecanismos permanecen más prolongadamente en el tiempo e incluso llegan a articularse a prácticas culturales de largo aliento; este es el caso de la Corporación Nasa Kiwe, principal institución de respuesta a la tragedia, la cual todavía funciona.

A partir de analizar y reconstruir la respuesta institucional y las percepciones sociales del riesgo y del desastre, en este artículo se sostiene que la Corporación Nasa Kiwe, creada bajo el gobierno de César Gaviria Trujillo (1990-1994) como principal estrategia de respuesta a la tragedia, se configuró a partir de la acción de distintos actores, principalmente: las diversas instancias nacionales $\mathrm{y}$ regionales del gobierno, la prensa y la población local. Es así como este mecanismo institucional de respuesta estuvo determinado por estos actores $\mathrm{y}$, tras su interacción, la respuesta 


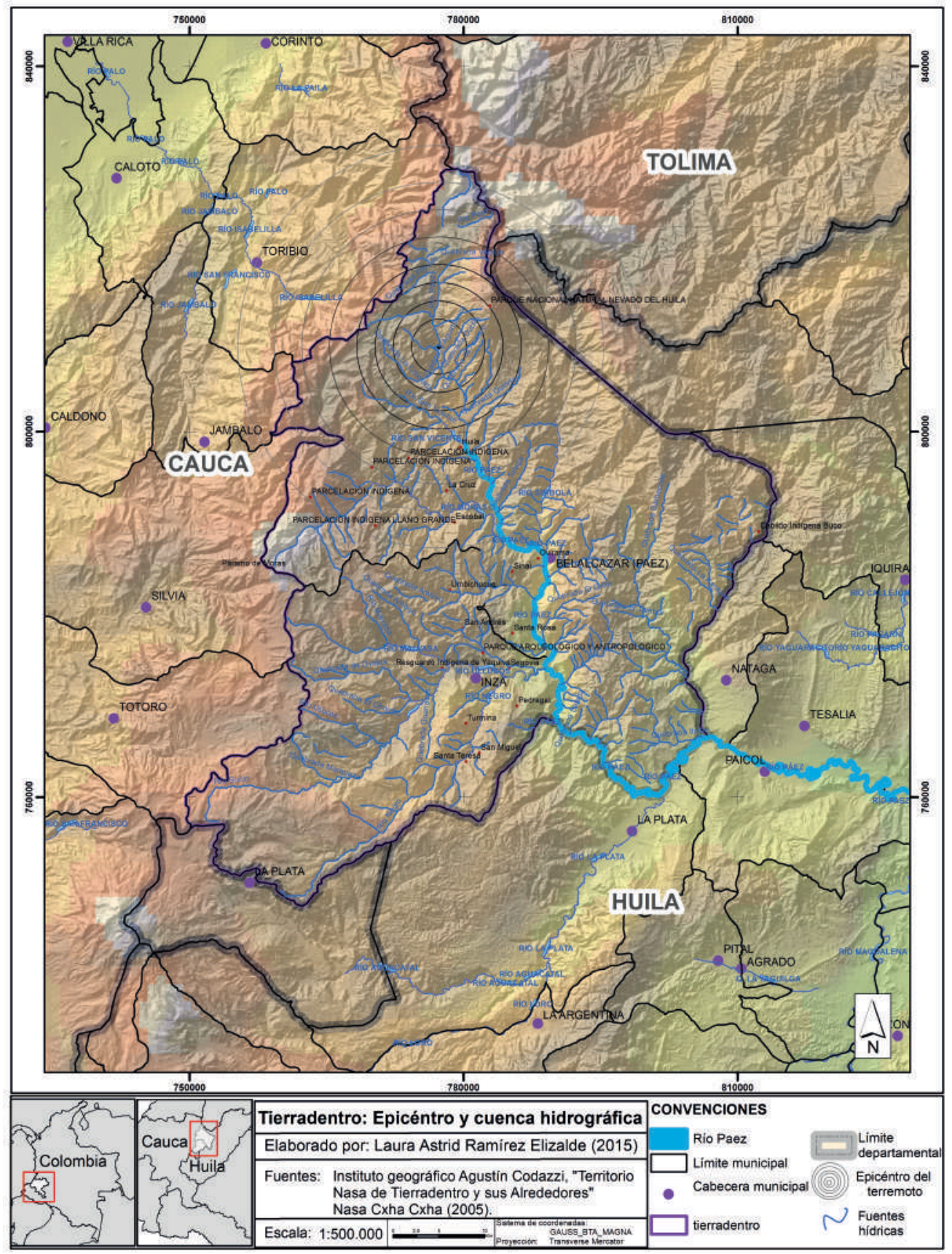

Figura 1: Mapa de epicentro del sismo y cuenca hidrográfica del río Páez 
institucional terminó por fundamentarse en políticas de discriminación positiva hacia la población indígena, lo cual se atempera con el clima político de la época y con el enfoque multiculturalista de la Constitución política colombiana de 1991 (VélezTorres, 2013).

Este artículo se organiza en cinco partes. Tras esta introducción, se presentan las consideraciones metodológicas, en las que, desde una propuesta interdisciplinar, se propone la articulación de métodos antropológicos, geográficos e históricos para la recolección de la información; en este sentido, las fuentes utilizadas reflejan la misma diversidad: se toman como fuentes primarias trabajos de cartografía social, observaciones etnográficas y documentos de archivo. El tercer apartado apunta a dar sentido a diferentes conceptos claves para comprender un evento adverso desde una perspectiva sistémica y relacional; esta sección teórica ayuda a entender cómo la participación social y la percepción social del desastre tienen un impacto en las acciones de respuesta frente a un desastre, a la vez que estas acciones tienen un efecto en la visión de la población frente al riesgo de una nueva ocurrencia; en el caso de Tierradentro, este carácter sistémico se expresará en cómo la institucionalidad, representada en la Corporación Nasa Kiwe, se ve en la necesidad de incorporar las percepciones sociales del desastre, en cuanto que el acaecimiento del desastre y la intervención institucional reconfiguraron aquello que las comunidades entendían como riesgoso.

Un cuarto apartado aborda las percepciones del desastre a partir de los imaginarios y de las representaciones que mediaron en la interpretación y en las acciones frente a la tragedia; acá se expone la manera como la interacción de diferentes actores llevó a que se negociaran dichas percepciones; es central el papel que en este capítulo cumplen la prensa, la presidencia, Ingeominas y la comunidad nasa; en esta sección se hace un énfasis especial en el surgimiento de la Corporación Nasa Kiwe, a fin de analizar cómo se constituye como respuesta institucional de carácter multicultural. Finalmente, a manera de conclusión, se enfatiza en cómo la dinámica social tras el desastre obligó al gobierno a actuar en coherencia con el principio de reconocimiento y protección étnica en Colombia, generando una respuesta institucional al desastre con clara orientación multiculturalista.

\section{Metodología}

Para dar cuenta de la respuesta institucional frente al desastre de 
Tierradentro y de cómo se articuló con la percepción social del desastre, se acuñó una metodología interdisciplinar que usó métodos etnográficos, de cartografía social y de revisión de fuentes de archivo. Esta perspectiva mixta permitió acceder a fuentes primarias que dan cuenta de las percepciones de los diversos actores, a la vez que ayudó a reconstruir históricamente la coyuntura político-institucional del desastre. A continuación se exponen los métodos y fuentes utilizados para dar cuenta de: 1) la respuesta institucional frente al desastre y 2) las percepciones sociales frente al desastre y al riesgo de repetición.

2.1. La reconstrucción y análisis de la respuesta institucional se elaboró a partir de tres grandes grupos de fuentes primarias:

\subsubsection{Se revisaron las discusiones del} Congreso de la República, Cámara y Senado, sobre la legislación de prevención y atención de emergencias, consignadas en Anales del Congreso (Diario oficial del Congreso de la República de Colombia). El periodo consultado inicia en 1986, momento en el que arrancan las discusiones en torno a la legislación de la atención y prevención de desastres, y finaliza en 1990, cuando se consolida el Sistema Nacional para la Prevención y Atención de Desastres. Esta consulta permitió elaborar un recuento histórico de la construcción de las políticas públicas nacionales en torno al tema de atención y prevención de emergencias, y comprender el marco normativo que el gobierno tenía para responder a la tragedia.

2.1.2. Se consultó el archivo institucional de la Corporación Nasa Kiwe, el cual sirvió para reconstruir la historia de la Corporación, sus estatutos y políticas; esta pesquisa también resultó útil para comprender la concepción gubernamental técnicocientífica sobre el desastre, en los estudios que adelantó Ingeominas en convenio con la Corporación. Del archivo de la Corporación resultaron de particular interés para esta investigación los siguientes documentos:

- Zonificación para usos del suelo en la cuenca del río Páez (Convenio Ingeominas-Nasa Kiwe), incluyendo la cartografía anexa a este informe, que responde a los estudios que terminaron por declarar la existencia de una zona de alto riesgo.

- Resguardo de Belalcázar (Convenio Nasa Kiwe- DRI, septiembre de 1996); consta de dos informes.

- Censo de Población CRIC-Nasa Kiwe en la zona del desastre, del 6 de junio de 1994- Análisis descriptivo de los principales indicadores de morbilidad $y$ mortalidad en los municipios 
de Páez e Inzá afectados por el desastre del 6 de junio (Convenio CRIC- Nasa Kiwe; abril de 1995).

- Herramientas de planeación para los planes de construcción. La región de Tierradentro, análisis general e impacto del desastre del 6 de 1994 (Convenio CRIC-Nasa Kiwe; mayo de 1995).

- Caracterización de la vivienda e impacto del desastre del 6 de junio de 1994 en la región de Tierradentro (Convenio CRIC-Nasa Kiwe; abril de 1995).

Esta revisión de archivo se adelantó en noviembre de 2011.

2.1.3. El tercer grupo de fuentes de archivo que permitió comprender la respuesta institucional corresponde a la revisión de prensa. Los medios de comunicación hicieron el seguimiento de las maniobras del gobierno para responder al desastre y documentaron los movimientos institucionales. Se consultó la prensa comprendida entre el 6 de junio y el 15 de agosto de 1994; se revisaron periódicos de circulación nacional, El Tiempo y El Espectador, y de circulación regional, El País y El Liberal.

2.2. Para acceder a la percepción social de los indígenas nasa sobre el desastre, quienes tenían visiones diferentes a los medios y al gobierno tanto sobre el desastre como sobre el riesgo y el territorio, se adelantó un trabajo etnográfico en el que se realizaron un total de ocho entrevistas: seis a líderes comunitarios que participaron activamente del proceso de reconstrucción tras la tragedia y dos colectivas (Ramírez, 2013). En este mismo contexto se adelantó un taller de cartografía social, en el que se proponía un ejercicio de representación del territorio antes y después de la tragedia; en estas representaciones se ilustraron diferentes elementos del entorno, entre ellos los riesgos y las amenazas. Como se enunció arriba, la percepción del desastre por parte de las instituciones se documentó con el trabajo de archivo.

\section{Conceptualización de riesgos y desastres}

Conceptos como amenaza, vulnerabilidad, riesgo y desastre han sido muchas veces definidos en relación con un carácter cuantificable, con lo que se busca medir y, ojalá, predecir el impacto de un fenómeno socio-natural en una escala poblacional concreta. En este artículo, sin embargo, se toman estos conceptos desde su dimensión cualitativa, con el objetivo de dar sentido a las condiciones de participación social de diferentes actores comunitarios, mediáticos y de gobierno en la respuesta frente al desastre de Tierradentro. Esta aproximación resulta apropiada para 
analizar la espacialidad del riesgo y del desastre desde una perspectiva político-institucional que, a través de una revisión histórica, reflexione sobre las respuestas que se gestan a nivel territorial para responder a eventos adversos.

En primer lugar, amenaza se entiende como cualquier elemento o evento capaz de generar un efecto adverso al ser humano; estos pueden ser de carácter natural, como terremotos, inundaciones o avalanchas, o pueden estar asociados al desarrollo y adquirir, de esta manera, un carácter antropogénico, como es el caso de la polución del agua, del aire o la contaminación electromagnética, entre otros. En términos de amenazas se contemplan, pues, no solo eventos de origen geo-físico, sino también factores de origen social potencialmente adversos a una población (Herzer, 2011).

En segundo lugar, por vulnerabilidad se entiende la propensión de una población a ser afectada por una amenaza. Según Cutter et al. (2008), el análisis de la vulnerabilidad se consolidó en la década de los noventa $\mathrm{y}$, aunque no existe una definición unificada, este giro interpretativo -de la amenaza a la vulnerabilidadconsistió en entender la adversidad en relación con las condiciones socioecológicas diferenciadas que afectan a una población o a un individuo.
Esta perspectiva se ha enfocado en explicar la interacción entre naturaleza y sistemas humanos, atendiendo a la edad, al género, a la etnicidad y a la clase social como determinantes de la exposición a los riesgos ambientales (Cannon, 1994; Spence et al., 2007). Pese a las ventajas que ha tenido esta perspectiva en la creación de acciones de prevención y mitigación frente a potenciales desastres (Cannon, 1994; Cutter, 1996; Cutter et al., 2003; Raschky, 2008), Furedi (2007, p. 487) afirma que también ha implicado una desventaja, ya que han sido pocas las investigaciones que buscan estudiar y comprender las respuestas efectivas en caso de ocurrencia de eventos adversos.

Desde una perspectiva local, varias investigaciones empíricas sobre vulnerabilidad han demostrado que el grado de preparación de una población frente a las amenazas varía en relación con su etnicidad y su estatus socio-económico (Spence et al., 2007). Así, la inequidad asociada a la posición social de un grupo implica que la exposición a un mismo riesgo resulte o no en una situación de desastre; esta posición particular es lo que configuraría la vulnerabilidad de ese grupo social. En concordancia, y desde una perspectiva global, que un país esté más expuesto a sufrir un desastre depende de la capacidad técnica, política y financiera del Estado de absorber los costos de prevención 
y mitigación del riesgo (Cannon, 1994, p. 26). Es por ello que puede afirmarse que los desastres a gran escala dejan menos víctimas e implican menores pérdidas económicas cuando mayor es la experiencia y mejor la estructura institucional del Estado para responder a estos eventos (Raschky, 2008).

En tercer lugar, riesgo se entiende como la probabilidad de que un evento adverso tenga lugar, y puede comprenderse como la relación entre amenaza y vulnerabilidad. Según muestra la Figura 2, la relación entre la amenaza y la vulnerabilidad genera una probabilidad de riesgo, pues es en la interacción entre los eventos potencialmente adversos y las condiciones socio-ecológicas particulares que se produce un riesgo determinado. Esa probabilidad de ocurrencia de un desastre es mediable o transformable con acciones de mitigación y prevención, como lo refiere el numeral 6 de la Figura 2.

Según García Acosta (2005), el riesgo es una construcción social que se da en dos niveles: primero, desde la construcción social de la vulnerabilidad, perspectiva según la cual las relaciones económicas, culturales y políticas aumentan la probabilidad de que una población sea afectada por un evento adverso, $\mathrm{y}$, segundo, desde la percepción del riesgo, sesgada o mediada por condiciones culturales, sociales y comunicativas. En concordancia, según Mary Douglas (citada por Elliott, 1983), es la cultura misma la que influye o enmarca ciertas situaciones -y no otras- dentro de la categoría de riesgo, volviendo este un concepto de valoración cultural y, por ello, polisémico.

En cuarto lugar, desastre se entiende como un evento socio-natural adverso; sin embargo, para ser más asertivos en el análisis de un caso concreto es necesario preguntar ien qué consiste dicha "adversidad"? Según la UNISDR y la Corporación OSSO (2013), las principales variables del análisis de un desastre son: (i) la pérdida de vidas humanas, (ii) la afectación a personas, (iii) la destrucción de viviendas destruidas y (iv) el daño a viviendas. A su vez, esta agencia llama desastre de carácter complejo a la combinación de eventos de tipo geológico, como un sismo, con eventos de tipo hidrometeorológico y climatológico, como lluvias, inundaciones y deslizamientos. Según esta caracterización, el evento de Tierradentro puede ser llamado "desastre", por sus numerosos muertos y daños, mientras su origen es de tipo complejo, al combinarse factores geológicos e hidrometeorológicos.

Aunque esta visión es ampliamente aceptada por entidades de gobierno y agencias de riesgo, no es la única. A través de un recorrido histórico sobre 
lo que se toma o no como "desastre", Furedi (2007) concluye que los eventos adversos son interpretados a través de un sistema de creencias que se encuentra, histórica y culturalmente, determinado. Esta propuesta construccionista tiene su fundamento en conceptualizaciones que, desde la sociología y la filosofía, concuerdan en afirmar que todo aquello que llamamos realidad está mediado por sucesos históricos, fuerzas sociales y aspectos ideológicos (Berger y Luckmann, 2001; Vélez-Torres, 2006; Hacking, 2001).

La perspectiva construccionista ha demostrado ser de gran utilidad en el estudio de riesgos y desastres, pues ha permitido ampliar la visión tecno-científica que dominó este campo del conocimiento hasta la década de los ochenta (Cárdenas, 1999). Hasta entonces, predominó la presunción del carácter natural de los riesgos y de los desastres, visión que negó las causas asociadas a acciones humanas y que llevó a centrar las políticas públicas y las prácticas de intervención en la predicción y atención técnica del daño (Cannon, 1994). Pero el construccionismo no solo permitió incorporar las acciones humanas dentro del análisis de los factores asociados a las amenazas, la vulnerabilidad, el riesgo $\mathrm{y}$, por supuesto, los desastres; también abrió el campo de comprensión de estos fenómenos al incorporar la percepción social del riesgo y de los desastres, y sugerir que un mismo evento puede ser interpretado $\mathrm{y}$ gestionado de formas completamente diversas entre actores sociales. El diagrama expuesto a continuación ilustra un sistema conceptual para la gestión del riesgo y desastres.

La Figura 2 expresa, precisamente, que las respuestas frente a un evento adverso dependen de las diferentes concepciones sobre lo que se interpreta como "adversidad" (ver numeral 4 de la Figura 2). A su vez, las acciones de respuesta son el resultado de la convergencia, diferencia y negociación entre diferentes actores. Por otro lado, este diagrama (Figura 2) evidencia que el desastre consiste en la materialización del riesgo y en el acaecimiento de las consecuencias adversas de un fenómeno o de un conjunto de fenómenos; en cambio, los conceptos de amenaza, riesgo y vulnerabilidad no se limitan a este carácter de "consecuencia", sino que aluden a las causas socio-naturales de un potencial desastre; estos tres conceptos son propicios, de esta forma, para el análisis y la gestión de eventos potencialmente adversos, mientras que el estudio de los desastres y de las acciones de reparación son pertinentes para analizar las respuestas multisectoriales frente a la ocurrencia de un evento adverso. Sin embargo, como muestra este diagrama, no hay una división tajante 


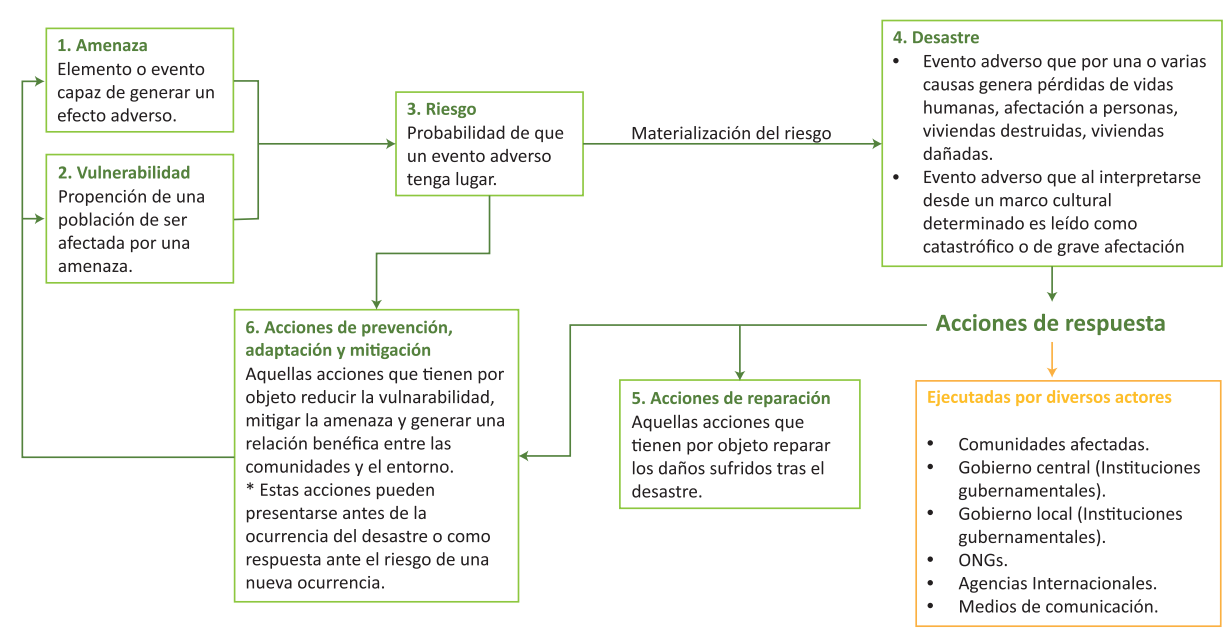

Figura 2: Sistema conceptual para la gestión de riesgos y desastres

entre los conceptos; por el contrario, en cuanto hacen parte de un sistema de gestión de riesgos y desastres, existen relaciones de dependencia y de retroalimentación.

Así, en la Figura 2 la retroalimentación se expresa en los numerales $5 \mathrm{y}$ 6 , donde se exalta que las acciones de reparación frente a un desastre tienen un impacto en nuevas acciones de prevención, adaptación y mitigación. A su vez, debido a que las acciones preventivas están dirigidas a impactar las amenazas y la vulnerabilidad $\mathrm{y}$, por ende, buscan modificar los factores o niveles de riesgo, también las acciones de reparación que han influido en nuevas acciones preventivas tendrán un efecto indirecto en la definición del riesgo. Como lo expresan Wachinger et al. (2013), junto a la confianza institucional, la experiencia frente a un desastre es determinante del accionar de un individuo ante el riesgo percibido. Así, del estado del arte construido por estos autores interesa resaltar su conclusión frente a la ventaja que representa la participación social y la cooperación entre diferentes sectores sociales e institucionales para mejorar un comportamiento preventivo $\mathrm{y}$, en últimas, garantizar una gobernanza del riesgo.

En concordancia con esta reivindicación de la participación social, Heijmans (2001) afirma que, pese al generalizado reconocimiento de que la población más vulnerable frente a desastres es también la más pobre, ha habido un descuido en comprender e incluir sus experiencias y visiones sobre los desastres. Los casos investigados por Heijmans (2001) ponderan el papel de la percepción de la población frente a la vulnera- 
bilidad, el riesgo y la amenaza, así como el impacto de la percepción en las acciones para prevenir o afrontar un desastre; en particular, el artículo destaca la manera como esta percepción social puede implicar caminos de acción diferentes a los esperados o establecidos por gobiernos y agencias de atención de desastres. El diagrama que se presenta a continuación, Figura 3, busca exponer las diferencias entre la participación de los actores en la gestión del riesgo desde un enfoque institucionalista y un enfoque con participación social.

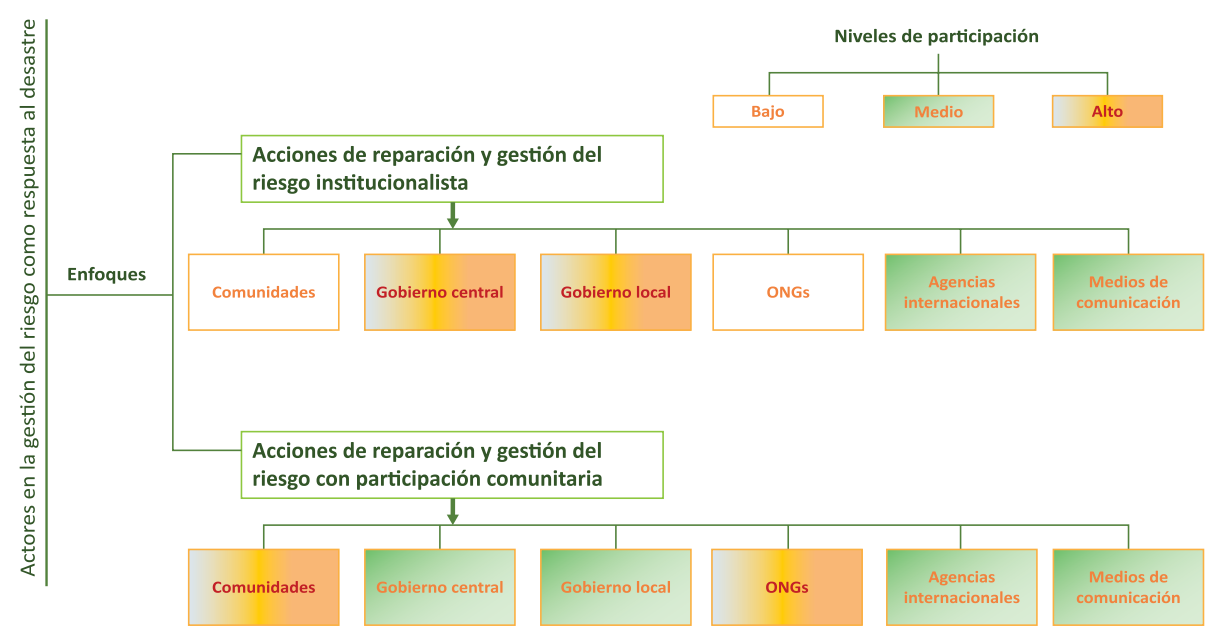

Figura 3: Enfoques en las acciones de reparación y en la gestión del riesgo

Si se tienen en cuenta los enfoques expresados en el Figura 3, es posible comprender las diferencias entre, por un lado, una ruta de acción institucionalista tradicional, en la que las visiones de comunidades y de las ONG no son relevantes para la definición de acciones frente a un evento adverso, y, por otro lado, una ruta de acción donde se exaltan la participación comunitaria y las visiones de estos actores sociales como elementos determinantes de las acciones frente a un evento adverso. En concordancia con este énfasis analítico sobre los actores y enfoques que median en las respuestas frente a desastres y la gestión de riesgos, a través del estudio de los casos del Terremoto de Limón, en Costa Rica, y de la contaminación del aire en México D.F., Bermúdez (1994) y Lezama (2004) muestran la necesidad de estudiar las relaciones sociales y políticas que surgen al mitigar un riesgo o responder frente a la ocurrencia de un desastre. De manera similar, a continuación se reconstruye la respuesta institucional 
en el caso de Tierradentro, la cual consistió, justamente, en un tránsito desde una ruta institucionalista hacia una ruta con amplia visión y participación de los indígenas nasa. A continuación se analiza cómo se dio este proceso de negociación entre actores sociales y políticos.

\section{Percepción social del desastre y acciones institucionales de respuesta}

El evento adverso y de características complejas en Tierradentro puso en tensión diferentes percepciones del "desastre", del territorio en el que ocurrió y del riesgo. Este apartado ilustra los imaginarios de ausencia estatal que marcaron los primeros días tras la tragedia, las críticas de la prensa y las respuestas frente al desastre gestadas por la presidencia de la República. Tras reconstruir las críticas de la prensa y contextualizar la actualidad institucional en la atención del desastre en Tierradentro, se muestra cómo el enfoque tecnocientífico, inicialmente utilizado por el gobierno, entró en tensión con la población afectada, por la forma de comprender, representar y planificar el territorio, el desastre y el riesgo. Finalmente, se analiza la creación de la Corporación Nasa Kiwe, como mecanismo de respuesta institucional que, con participación comunitaria, intentó mediar entre los diferentes actores y crear una respuesta al desastre con enfoque diferencial.

\section{La prensa y su presión sobre presidencia}

A finales de 1986, después de la avalancha de Armero $^{7}$, se crea en Colombia la Oficina Nacional de Atención de Emergencias -ONAE-; posteriormente, en 1987, César Gaviria, como Ministro de Gobierno del presidente Virgilio Barco (19861990), presentó ante el Congreso el Proyecto de Ley 124, que organizaría de manera centralizada el sistema para la prevención y atención de emergencias. En 1988, la ONAE pasó a ser parte de un Sistema Nacional para la Prevención y Atención de Desastres -SNPAD-, a través de la Ley 46 de 1988, que, al reglamentarse en mayo de 1989, crea el sistema como una dependencia directa de la Presidencia de la República. En 1990, bajo la administración Gaviria, la ONAE es adjudicada al Ministerio de Gobierno, bajo el nombre Dirección Nacional para la Prevención y Atención de Desastres DNPAD- (Stuart Olson et al., 1995). Desde el inicio se trató de un sistema que dependía administrativamente del nivel central; no obstante, en 1988 se le asignó, entre otras, la responsabilidad de establecer comités

\footnotetext{
7 El 13 de noviembre de 1985, la erupción del volcán Nevado del Ruíz, en el departamento del Tolima, generó cuatro corrientes de lodo que descendieron por las laderas del volcán a más de $60 \mathrm{~km} / \mathrm{h}$ y causaron la muerte de al menos 23.000 personas (ver: Olson y Sarmiento, 1995).
} 
regionales y locales de respuesta y prevención de desastres (Zeiderman y Ramírez, 2010).

En junio de 1994, pese a la existencia de este sistema nacional, la respuesta institucional que se dio en los primeros días al desastre de Tierradentro estuvo caracterizada por una clara desconexión entre la acción de los organismos regionales, la DNPAD y la presidencia. Mientras hubo problemas en los flujos de información entre estas instancias de gobierno, la falta de acción de los comités y de las autoridades locales limitó la respuesta de las instituciones regionales del Cauca y del Huila. De esta forma, la coordinación de la respuesta se centró en Bogotá (Ramírez, 1995); este enfoque centralista en la toma de decisiones constituyó una gran barrera a la hora de emprender acciones de atención efectivas.

La tragedia se presentó en un contexto político marcado por el cierre de gobierno de César Gaviria, quien se vio obligado a responder de inmediato sin saber con exactitud qué había pasado, cuál era el área afectada y cuántas personas había perjudicadas. No había censos de la población, ni conocimiento del número de asentamientos ubicados en el sector, lo que, junto a las características geofísicas del terreno, dificultó las labores de rescate y generó una fuerte crítica en la opinión pública.
A mediados de 1994, cuando ocurrió el terremoto, Gaviria se encontraba próximo a entregar su cargo con un alto nivel de favoritismo, por lo que una mala respuesta podría, con facilidad, opacar su imagen en los últimos días de mandato (Cardona, 1995; Ramírez, 1995; Wilches, 1995).

La prensa expresó una fuerte crítica al gobierno por el desconocimiento de la zona y de su población. Los periódicos y noticieros rápidamente se llenaron de imágenes de niños enlodados y de familias devastadas que reafirmaban un imaginario de los territorios indígenas como zonas aisladas, pobres y donde el Estado era ausente. Los periodistas fueron quienes primero lograron ingresar a varios de los sectores afectados, por lo que se encargaron de documentarle al gobierno lo que ellos consideraban la magnitud del desastre; a la vez, las instituciones encargadas del rescate aún tenían dificultades para llegar a la zona y organizarse articuladamente. Un comportamiento similar mostraron los medios de comunicación frente al terremoto de Limón, en Costa Rica (Bermúdez, 1994): un cuadro de ineptitud estatal se dispersó y la crítica recayó directamente en la falta de prevención y de respuesta del gobierno. Cinco días después del siniestro, la prensa colombiana se concentraba agudamente en las críticas; en uno de los periódicos de 
circulación nacional podía leerse:

La catástrofe de Tierradentro, infinitamente más grave de lo que al principio pareció, demuestra una vez más cómo está el país de impreparado para prevenir riesgos y acudir en socorro inmediato de las víctimas. El hecho de no haber funcionado las alarmas instaladas en las vecindades de Belalcázar [Capital del departamento de Páez en Tierradentro], así como la demora en activar los mecanismos de la pomposa Oficina de Prevención y Atención de Desastres de la Presidencia de la República, revelan que poco o nada aprendimos de la dramática devastación de Armero (El Tiempo, 11 de junio de 1994).

Esta crítica fue generalizada y responsabilizó al gobierno de adjudicarle la atención de este tipo de emergencias a un organismo centralizado con poca maniobra a nivel regional, como lo era la presidencia; sin embargo, desde presidencia se responsabilizó al sistema como si se tratara de un organismo independiente. En el encabezado de un artículo del 12 de junio, en el periódico El Tiempo se lee:

Cuentan que el presidente Gaviria regresó muy bravo de su visita a la zona del desastre. Y que el ministro Pardo, también muy furioso, destituyó a gerentes regionales del Idema y a directores de la Defensa Civil por incompetencia y burocratismo. $\mathrm{Su}$ rabia es explicable. También su desconcierto (12 de junio de 1994).

En el mismo artículo, después de enunciar el descontento presidencial, es evidente cómo la crítica ya no recae sobre la responsabilidad de la presidencia, sino directamente sobre la DNPAD (antes ONAE).

No hubo reacción inmediata ante la crisis. Tampoco unidad de mando, ni centralización de la información. No apareció autoridad nacional alguna que actuara a tiempo y asumiera el control. Al otro día, en la Oficina de Prevención de Desastres (qué tal el nombrecito) ni siquiera atendían. Solo hacia mediodía se dieron cuenta de la gravedad de los hechos, cuando ya los medios de comunicación estaban manejando la emergencia ( $E l$ Tiempo, 12 de junio de 1994).

A su vez, la DNPAD respondió a las críticas adjudicando la responsabilidad a los organismos regionales y evidenciando las fracturas del sistema de atención y prevención de desastres. Si bien la DNPAD tenía la función de administrar, coordinar y controlar que las actividades de respuesta frente al desastre se desarrollaran de manera eficaz a través de comités 
regionales y locales, esta estrategia demostró ser más una visión ideal que una estructura de atención efectiva. A la hora del siniestro, no había instituciones regionales creadas que soportaran adecuadamente este sistema piramidal, por lo que la única unidad de respuesta real fue la Dirección en Bogotá. La tensa situación entre las distintas escalas del sistema de prevención fue denunciada en un artículo titulado "Comités regionales tampoco respondieron ante la tragedia", publicado el 17 de junio del mismo año por El Tiempo. Aunque el sistema dependía de Presidencia (Ministerio de Gobierno), Gaviria fue hábil al enfocar sus anuncios públicos en reprochar la acción de los organismos regionales y del sistema de prevención, y en prometer una reparación a las víctimas.

Gaviria reiteró que si bien al comienzo de la tragedia no se reaccionó con prontitud, como se hubiera querido, a las pocas horas se introdujeron correctivos y se ha hecho un enorme esfuerzo para que haya una atención oportuna (El Tiempo, 30 de junio de 1994).

El anuncio de Gaviria de enmendar la situación estaría anclado en la creación de un órgano institucional que respondiera al carácter étnico de las comunidades afectadas y a la necesidad de una "reparación histórica”. Este mensaje público perfila el giro que dio el gobierno desde una respuesta de carácter institucionalista hacia una respuesta que, con perspectiva diferencial, atendería a la historia y otras particularidades culturales de la población afectada.

\section{Entre la representación tecno- cientificista de Ingeominas y la visión étnica del territorio}

En esta sección se contextualiza uno de los elementos centrales en la atención institucional del desastre y en la creación de la Corporación Nasa Kiwe: el encuentro entre dos formas de comprender el territorio y, por ende, de interpretar el desastre y percibir el riesgo. Como en el caso del desastre de Limón, en Costa Rica (Bermúdez, 1994, p. 221), aquí también se expresa una mezcla entre las creencias tradicionales de la población local y el conocimiento técnico, transmitido por instituciones y medios de comunicación. En el caso de Tierradentro, la respuesta inicial del gobierno implicaba una representación técnica del espacio y del riesgo, en la cual tuvo un papel importante Ingeominas -institución encargada de hacer los estudios técnicos del suelo-. A esta visión institucional se contrapone la visión del territorio de quienes lo habitaban, donde la concepción de desastre, como veremos, estuvo mediada por 
su experiencia de territorialidad (Agnew, 1994).

Tras el desastre, la reubicación de alrededor de 7.000 personas tuvo como criterio técnico un estudio de Zonificación para usos del suelo en la cuenca del río Páez que, junto a una cartografía de zonificación elaborada por Ingeominas, sirvió de base al gobierno para declarar gran parte del área afectada como zona de alto riesgo (ver Ramírez, 2013 e Ingeominas, 2005). Esta representación científico-técnica del territorio llevó a que el gobierno decretara la reubicación basándose en un discurso desde el que se comprendió el territorio, a partir de un análisis geofísico, como un espacio con dinámicas predecibles. Para el gobierno y sus instituciones, el riesgo parecía una variable cuantificable que evaluaba la propensión a terremotos, deslizamientos y avalanchas.

Pero la imagen del territorio en las comunidades afectadas distaba mucho de la representación de Ingeominas. Para los indígenas, la mayor expresión de "riesgo" era la pérdida del territorio tradicional y la posibilidad de que se interrumpiera la continuidad cultural nasa; por esta razón, fue la noción técnica del riesgo, en la que se fundamentaba el criterio de reubicación, la que se convirtió en amenaza para la comunidad indígena afectada. En respuesta, entre los indígenas creció una fuerte resistencia a comprender su territorio como una zona de alto riesgo.

Los nasa tienen una visión muy distinta de las avalanchas, los deslizamientos y los terremotos, pues los elementos diferentes a lo humano -como los ríos, las montañas y los rayos- se consideran seres vivos $\mathrm{y}$ actores sociales claves. De las avalanchas, por ejemplo, han nacido personajes importantes en la lucha histórica de los nasa; este es el caso del líder Juan Tama ${ }^{8}$, quien, siendo hijo de la laguna, del trueno y de la estrella, en su nacimiento bajó en una avalancha. Estas representaciones míticas de la tormenta y del crecimiento de las aguas demuestran que Tierradentro es un territorio en el que llueve, tiembla, hay deslizamientos, derrumbes y avalanchas. El volcán, la lluvia, los ríos, las lagunas y las montañas tienen un papel activo en el territorio; en consecuencia, son actores claves de la organización social y de la regulación de la vida cotidiana.

La percepción que antes de la tragedia tenían los nasa sobre los riesgos y

\footnotetext{
8 Juan Tama no es solo un ser mítico, también es un personaje histórico. En la Historia Política de los Paeces, Víctor Daniel Bonilla narra la vida del líder indígena, afirmando que fue el más importante cacique de Vitoncó y de todo el pueblo Paez, pues fue por medio de él que los indígenas obtuvieron los títulos de los resguardos con los que históricamente han defendido su territorio (Bonilla, 1980, p. 15-17).
} 
las amenazas dista de la concepción técnico-científica; así lo revelan los resultados de un taller con indígenas de esta región, adelantado el 21 de octubre de 2011, donde se indagó por la percepción de los riesgos y amenazas que existían en Tierradentro al momento de ocurrir el siniestro y tras el proceso de reasentamiento que afrontaron algunos.

Según lo manifestaron los participantes, las amenazas antes del evento tenían que ver con la pérdida cultural y los conflictos sociales asociados al narcotráfico, la guerrilla, la medicina y la religión católica. En este taller se instruyó a los participantes para pintar el territorio e identificar en él riesgos y amenazas en orden jerárquico; sin distinguir entre riesgos y amenazas, los participantes ubi-caron como principal amenaza la subversión, en tanto parte del territorio padecía desde entonces el conflicto armado; en segundo lugar se identificó como amenaza la existencia de cultivos ilícitos, que se relacionaron con la pérdida cultural, pues el "dinero fácil" que trajo el cultivo y la producción de amapola alteró las dinámicas comunitarias y las prácticas culturales, cambiando, principalmente, la forma de vestir y los hábitos alimenticios; la tercera amenaza correspondió a la "medicina occidental", en tanto amenazaba con desplazar la medicina propia y las prácticas curativas tradicionales; en el cuarto lugar se ubicó la religión, y, finalmente, cerrando la lista se situó el "suceso del terremoto". Interesante resultó que el desastre ganó este quinto lugar, no tanto por los numerosos muertos y daños físicos, sino por la reubicación del $30 \%$ de la población; es decir, es la fragmentación comunitaria y la potencial pérdida cultural la que se considera una amenaza, y no el factor geofísico valorado por Ingeominas.

Según Gómez y Ruiz (2000), el equilibrio social nasa fue alterado en los primeros años de la década de los noventa, cuando en algunos resguardos se introdujo el cultivo intensivo de amapola. La bonanza económica producida por "la mancha", como se le llamaba a esta planta, incentivó su cultivo, incluso en territorios sagrados, lo que desequilibró el uso tradicional de la tierra y la "armonía con la naturaleza". Esta situación, además de traer un fuerte impacto ambiental, pues se deforestaron los páramos y bosques de la región al ampliarse la frontera agrícola, generó un conflicto social alrededor del "dinero fácil" y dio origen a riñas en las comunidades. Así lo explicó un indígena nasa en una entrevista en octubre de 2012:

Los médicos tradicionales dicen que eso [el desastre] fue un llamado de atención de la Madre Naturaleza. ¿En qué sentido? (...) [por] la tala de bosques, quemas, pues más por el lado del cultivo ilícito que en ese 
entonces estaba en buen momento. Entonces, la gente por avanzar con sus cultivos ilícitos estaba talando la mayor parte de los bosques. Había una descomposición social, prácticamente del resguardo de Mosoco, bueno y otros también casi que estaban en las mismas situaciones (...) Se movían recursos de los cultivos ilícitos y los muchachos a muy temprana edad pasaban tomando sus tragos, haciendo sus desórdenes, no había orden y había muchas disputas, problemas entre los mismos vecinos, discusiones, discusiones que ya no sólo se iban a alegato, sino que ya sacaban cuchillos, armas de fuego. Bueno, la verdad es que se estaba volviendo algo incontrolable que ya la misma autoridad tampoco podía ejercer su autonomía. Entonces, los mayores miran y dicen que, tal vez, la Madre Naturaleza por eso llamó (Ramírez, 2013).

El antecedente histórico de la amapola cumplió un papel fundamental en la percepción de los nasa frente al desastre, pues este cultivo y la cultura del dinero fácil se explicó como una causa, tan cultural como ambiental, del evento adverso desencadenado por el terremoto. Para ellos, parte de la explicación del desastre está asociada a este cultivo, por lo que les resultó comprensible que el terremoto y la avalancha hubiera afectado principalmente a los sectores con cultivos más extensos. Así, el desastre, sin perder su carácter de tragedia por el dolor que trajo la muerte de los familiares, ha sido entendido también como un proceso que permitió la limpieza del territorio y la reconfiguración de los lazos sociales 9 . Como se muestra a continuación, el choque que se presentó entre estas formas disímiles de comprender el territorio fue lo que impulsó la creación de la Corporación Nasa Kiwe.

\section{Creando convergencias entre dife- rentes percepciones del desastre: el surgimiento de la Corporación Nasa Kiwe}

Tres días después del desastre, el presidente Gaviria expidió el Decreto 1178 de 1994, por medio del cual se declaró el Estado de Emergencia, se informó sobre la insuficiencia del Sistema Nacional de Atención y Prevención de Desastres para atender la calamidad y se estableció la necesidad de crear un organismo para enfrentar la crisis, lograr la reconstrucción y rehabilitar la zona. En el marco de esta nueva normativa se creó la Corporación Nasa Kiwe, que tuvo como función coordinar la reconstrucción y reubicación de la población afectada y articular las

\footnotetext{
9 Para entender cómo se organizó el proceso de reubicación de las víctimas, y cuáles fueron algunas de las oportunidades y dificultades vividas por las comunidades indígenas reasentadas ver Ramírez (2013).
} 
instituciones estatales. La prensa exaltó la intención del Presidente de concertar con los nasa:

Los 500 años de discriminación que hubo en América y Colombia, agregó el presidente Gaviria, se van a remplazar por unas décadas de cooperación y trabajo conjunto. La Corporación no solo será un escenario de reconstrucción, sino de concertación, afirmó al ratificar su solidaridad y compromiso con los cerca de 17.000 damnificados. La ocasión infausta tendrá que servir, dijo Gaviria, de desafío para demostrar que Colombia está cambiando y que somos capaces de ser solidarios (El Tiempo, 30 de junio de 1994).

Fue la creación de la Corporación la acción de reparación más importante para responder a la crisis, acción en la cual se lee el giro del gobierno hacia la participación de actores comunitarios y de ONG, a la vez que se expresa claramente la racionalidad multiculturalista del Ejecutivo. A través de su apuesta por una "reparación histórica", el gobierno superó su imagen de ineficiente, para proyectarse como un gobierno garantista, lo cual generó un ámbito de confianza en el proceso de reconstrucción.

La idea de Gaviria de crear una nueva Corporación, en lugar de fortalecer las estructuras existentes, generó respaldo mediático, pero también inconformidad entre las personas que lideraban el tema de manejo de riesgos en el país. Gustavo WilchesChaux, quien había participado en la conformación de la Red Latinoamericana de Prevención y Atención de Emergencias (La RED), escribió un comunicado criticando fuertemente esta decisión. Argumentó que la postura del gobierno era inadecuada, porque en un principio el decreto que creaba la nueva Corporación no tenía en cuenta las particularidades socioculturales y ambientales del sector y no vinculaba a la comunidad en el proceso de recuperación (Wilches-Chaux, 2000).

Con esta crítica, Wilches-Chaux entró en el debate político; de hecho, tras sus declaraciones, el entonces Ministro de Defensa, Rafael Pardo, lo llamó para informarle que el gobierno ya había creado la Corporación, que en un primer momento se llamó Corpopaeces $^{10}$, y que él había sido escogido como director. Esta selección fue, una vez más, una inteligente estrategia política. Como Director Regional del Sena en el departamento del Cauca, Wilches-Chaux había liderado, en 1983, los procesos comunitarios de reconstrucción tras el terremoto de Popayán. Además de tener un alto conocimiento del tema, tenía un reconocimiento importante en

10 Los indígenas nasa han sido conocidos históricamente como paeces; nasa es su autodenominación. 
la región, y su designación permitiría mantener en la práctica el vínculo con el Sistema Nacional de Prevención y Atención de Desastres, algunos de cuyos miembros también hacían parte de La RED (Wilches-Chaux, 1993). Wilches-Chaux decidió aceptar la dirección de la Corporación, y su primera actividad consistió en cambiar el nombre, que pasó de Corpopaeces a Corporación Nasa Kiwe: Nasa, que significa gente, todo lo que tiene vida, y Kiwe, que significa tierra, territorio (Gómez y Ruiz, 2000, p. 24).

Gaviria terminó su mandato con una imagen favorable en agosto de 1994, dos meses después de la tragedia. Aunque ya no fue primera plana, la Corporación continuó siendo fundamental en la interacción de las comunidades con el Estado. La propuesta administrativa de Wilches-Chaux, quien permaneció en la dirección de la Corporación durante dos años, partió de vincular a las comunidades al proceso de reconstrucción y reasentamiento; esto permitió que el equipo de la nueva institución tuviera una alta participación de líderes indígenas que, además de conocer la zona, tenían comunicación directa con los afectados y manejaban la lengua y la cosmovisión. Este enfoque de inclusión se evidencia en los objetivos de la Corporación, en los que se plantea:
Coordinar en asocio con la Dirección Nacional para la Prevención y Atención de Desastres (DNPAD), el diseño y ejecución de un "Plan general de recuperación y desarrollo sostenible de la zona afectada", y garantizar la real participación y capacidad de decisión de las comunidades $y$ de sus instancias representativas en las distintas etapas. Para el efecto la Corporación tendrá en cuenta la diversidad étnica y cultural existente en la zona, la cual surge de la existencia de comunidades indígenas de distintos grupos étnicos y de comunidades mestizas y negras de características urbanas y rurales (Wilches, 2000, p. 30).

Esta metodología participativa e incluyente, además de estar en concordancia con la nueva Constitución, también partió de una perspectiva en la que se valora la visión y participación social como una variable determinante de las acciones de reparación y de la gestión del riesgo. A su vez, la Corporación interpretó el desastre como el resultado de la falta de prevención en el contexto de una convivencia inadecuada entre los sujetos y el entorno natural (Serna Quintana, 2010). Esta visión coincide con la perspectiva construccionista abordada en el marco conceptual, 
según la cual se reconoce la antropogénesis de la vulnerabilidad y la importancia de incluir a los actores comunitarios en la gestión de los desastres y del riesgo frente a una potencial nueva ocurrencia.

\section{Discusión y conclusiones}

El sismo y las posteriores avalanchas en Tierradentro, en junio de 1994, constituyeron un evento adverso complejo, que dejó más de 1.100 personas muertas y cerca de 7.000 reubicadas. Entonces, César Gaviria se encontraba en sus últimos días como presidente de Colombia, y las críticas frente al accionar de su gobierno en respuesta a la tragedia amenazaban con opacar su gestión en el Ejecutivo. La necesidad del presidente Gaviria de responder a la crisis a través de decisiones de gobierno que resultaran coherentes con los postulados de la reciente Constitución Política de 1991 generó un ambiente propicio en el que la respuesta del gobierno viró, desde el cientificismo de Ingeominas, hacia una atención con participación social y perspectiva indigenista materializada en la creación de la Corporación Nasa Kiwe. Este giro implicó una negociación de visiones frente al territorio, al desastre y al riesgo de una nueva ocurrencia; en esta negociación, la percepción social de los indígenas jugó un rol determinante.
En este artículo se ha reconstruido el desastre a partir de la forma en la que diferentes actores y visiones interactuaron en tensión y terminaron por moldear unas acciones de respuesta, tanto en el campo de la reparación de los daños sufridos tras el desastre, como en el campo de la prevención del riesgo frente a una potencial nueva ocurrencia. La propuesta conceptual adelantada reconoce relaciones sistémicas entre los términos vulnerabilidad, amenaza, riesgo y desastre; en particular, muestra las relaciones de dependencia y de retroalimentación que surgen cuando las acciones de reparación frente a un desastre impactan las nuevas acciones de prevención, adaptación y mitigación; o, también, cuando las acciones preventivas, dirigidas a impactar las amenazas y la vulnerabilidad, generan un efecto indirecto sobre la definición del riesgo. Estas relaciones se complejizan al detallar el rol que juegan diferentes actores sociales y políticos en la definición de acciones frente a un desastre.

En el caso de Tierradentro, este artículo ha analizado la dimensión político-institucional histórica de respuesta al desastre a través de la caracterización de las diferentes percepciones y acciones que intervinieron en su gestión. La espacialidad del riesgo y del desastre se analizó a partir de tres ejes; el primero indagó por la interacción entre la Presidencia 
y la prensa, y permitió ver cómo el discurso de atención a las víctimas, que apeló al carácter multicultural manifiesto en la Constitución, se generó como respuesta del Ejecutivo a las fuertes críticas de la prensa y de la opinión pública; el segundo eje se centró en la disyuntiva existente entre las visiones del territorio de Ingeominas y de los indígenas nasa, y el tercer eje analizó el carácter indigenista de la creada Corporación Nasa Kiwe, que fue resultado del agitado panorama nacional, empapado por un proceso constituyente $\mathrm{y}$ cobijado por una nueva Constitución Política de carácter multicultural.

En el caso de la percepción indígena del evento, se encontraron dos rasgos centrales de la cosmovisión nasa que mediaron esta visión: en primer lugar, los nasa entendieron este evento como un episodio de muchos previos; de hecho, y como se desarrolló en el acápite anterior, la mitología nasa habla de cómo importantes líderes, como Juan Tama, han surgido, precisamente, en eventos como las avalanchas; así las cosas, el evento que tuvo lugar en la zona no fue ajeno a los conocimientos y creencias de la comunidad. En segundo lugar, para los habitantes de la zona, el evento debía explicarse en relación con el cultivo ilícito de amapola, que se había extendido en la región y que, según la comunidad, constituía una verdadera amenaza para la continuidad cultural de los nasa de Tierradentro. Desde esta perspectiva, el "desastre" lo era por los muertos y por las viviendas y cultivos perdidos, pero de alguna manera constituía también una limpieza forzosa $y$ necesaria del territorio.

Por otro lado, para los nasa el riesgo estaba dado por la posibilidad de ser reubicados y perder su territorio, más que por la ocurrencia de un nuevo "desastre", como lo pronosticó Ingeominas. De hecho, lo que el análisis de este caso permite observar es que esta diferente percepción del desastre, por parte del gobierno central, en cabeza de Ingeominas, y de las comunidades, fue un factor decisivo en la respuesta de la Presidencia de crear la Corporación Nasa Kiwe. En las acciones de esta entidad encontramos un claro eco de la tendencia construccionista de entender el riesgo en su dimensión sociológica, desde el análisis de las condiciones de vulnerabilidad de la sociedad local. A la vez, la Corporación se distanció un poco de la interpretación cientificista y naturalista del desastre -encarnada por Ingeominas- y entró a valorar también las condiciones históricas de vulnerabilidad de la población indígena afectada $\mathrm{y}$ las visiones particulares sobre el territorio, el desastre y el riesgo futuro que percibían los indígenas nasa. 
La visión indigenista y de largo plazo que sostuvo la Corporación puede ser leída como un logro de las organizaciones sociales que participaron en la Constituyente y que apelaron a la necesidad de generar acciones afirmativas para la población étnica. También puede leerse como el resultado de debates y luchas institucionales de organizaciones que, como La RED, buscaron promover en América Latina lecturas más completas y complejas sobre la relación entre los sujetos sociales y sus entornos naturales. La respuesta del Ejecutivo frente a la tragedia fue estratégica, al diseñar, desde una política de discriminación positiva, un mecanismo de atención a la tragedia que sorteó el desconocimiento que el gobierno central tenía sobre la población y sobre la zona afectada. Así, a la vez que la Corporación se propone incorporar las visiones de los indígenas y promover procesos de participación social en la reparación a la población afectada, también se presenta como una apuesta política por diseñar una institución que respondiera al desastre desde las particularidades de la población étnica afectada. En consecuencia, la necesidad de reconocer las particularidades sociales y culturales de la población dio al desastre un carácter de laboratorio político y social del multiculturalismo, a través del diseño institucional para la atención del desastre y para la gestión del riesgo.

\section{Referencias}

Agnew, J. (1994). Territory. En: Johnston, R.J., Gregory, Derek y Smith, David (Ed.), The Dictionary of Human Geography, $3^{\circ}$ ed. Cambridge: Basil Blackwell.

Berger, P. y Luckmann, T. (2001). La construcción social de la realidad. Buenos Aires: Amorrortu.

Bermúdez, M. (1994). El manejo institucional y percepción de la población en el terremoto de Limón. Revista Geol. Amér. Central, vol. Especial Terremoto de Limón, 221-224.

Cannon, T. (1994). "Vulnerability analysis and the explanation of "natural' disasters". In: Varley, Ann (ed.), Disasters, development and environment. New York: John Wiley \& Sons Ltd. Pp. 13-30.

Cárdenas, C. (1999). Evolución y perspectivas del tratamiento de los riesgos socionaturales en Colombia. Charla dictada en la Sociedad Geográfica de Colombia, agosto de 1999, Colombia.

Cardona, D. (1995). El sismo del 6 de junio de 1994. Revista Desastres y Sociedad, 4, 19-32. Recuperado de: http://www.desenredando.org/public/revistas/dys/.

Cutter, S. (1996). Vulnerability to environmental Hazards. Progress in Human Geography, 20(4), 529-539. 
Cutter, S.; Barnes, L.; Berry, M.; Burton, C.; Evans, E.; Tate, E. y Webb, J. (2008). A place-based model for understanding community resilience to natural disasters. Global Environmental Change, 18, 598-606. ISSN: 0959-3780.

Cutter, S.; Boruff, B. y Shirley, L. (2003). Social vulnerability to environmental hazards. Social science quarterly, 84(2), 242-261.

DNP (2004). Evaluación de Riesgos Naturales Colombia. Estudio sobre desastres en Colombia: Estimiación de pérdidas y cuantificación de costos. Bogotá: Departamento Nacional de Planeación.

Elliott, D. (1983). Risk and Culture: An Essay on the Selection of Technical and Environmental Dangers. Faculty Scholarship Series, Paper 2192, 888-899. Recuperado de: http:// digitalcommons.law.yale.edu/fss_papers/2192.

El Tiempo (11 de junio de 1994). Tratamiento de las emergencias. Bogotá.

El Tiempo (12 de junio de 1994). Cuando la tierra ruge... Bogotá.

El Tiempo (30 de junio de 1994). Se hará justicia a los paeces: Gaviria. Bogotá.

Furedi, F. (2007). The changing meaning of disaster. Area, 39(4), 482-489.

García Acosta, V. (2005). El riesgo como construcción social y la construcción social de riesgos. Desacatos, 19, 11-24.

Gómez, H. y Ruíz, C. A. (2000). Los paeces: gente territorio, metáfora que perdura. Popayán: Universidad del Cauca.

Hacking, I. (2003). ¿La construcción social de qué? Barcelona: Paidós.

Heijmans, A. (2001). 'Vulnerability': a matter of perception. Disaster Management Working Paper 4. Benfield Greig Hazard Research Centre.

Herzer, H. M. (2011). Construcción del riesgo, desastre y gestión ambiental urbana. Perspectivas en debate. Revista Virtual REDESMA, 5(2), 51-60. Recuperado de: http://www.revistasbolivianas.org.bo/pdf/rvr/v5n2/a08.pdf

Ingeominas (2005). Zonificación para usos del suelo en la cuenca del río Páez. Unidad Operativa Popayán, Observatorio vulcanológico y sismológico.

Lavell, A. (2005). Formas de construcción social de un desastre: El caso del huracán Mitch en Centroamérica. En: Fernández, A. (Comp.), Comarcas vulnerables: riesgos y desastres naturales en Centroamérica y el Caribe. Pp. 11-44. Buenos Aires: CRIES.

Lezama, J. L. (2004). La construcción social y política del medio ambiente. México D.F.: Colegio de México.

Oslon, R. y Sarmiento, J. P. (1995). El desastre de Cauca y Huila en Colombia no es otro Armero. Revista Desastres y Sociedad 4, 7-18. Recuperado de: http://www. desenredando.org/public/revistas/dys/

Pachón, X. (1996). Los nasa o la gente paez. En: Geografía humana de Colombia. Región Andina Central, Tomo VI, Vol. II. Bogotá: Banco de la República.

Ramírez, F. (1995). El sismo de Páez, respuesta y manejo de un desastre. Revista Desastres y Sociedad, 4, 98-130. Recuperado de: http://www.desenredando.org/public/revistas/dys/ 
Ramírez, L. (2013). ¿Irse, quedarse o llevar el territorio a cuestas? El proceso de reorganización territorial nasa después del terremoto de 1994 en Tierradentro, Cauca. Tesis de Maestría en Geografía Humana, Universidad de los Andes, Bogotá.

Raschky, P. A. (2008). Institutions and the losses from natural disasters. Natural Hazards Earth System Sciences, 8, 627-634.

Serna Quintana, C. (2010). ¿Naturales o naturalizados? Una aproximación histórica a los desastres asociados a inundaciones y deslizamientos en Medellín, 1930-1991. Tesis de pregrado en Historia, Universidad de Antioquia.

Spence, P.; Lachlan, K. y Griffin, D. (2007). Crisis Communication, Race, and Natural Disasters. Journal of Black Studies, 37, 539-554.

UNISDR y Corporación OSSO (2013). Impactos de los desastres en América Latina y el Caribe, 1990-2011. Tendencias y estadísticas para 16 países - Informe.

Vélez-Torres, I. (2006). Inconmensurabilidad conceptual: el caso de la hoja de coca en uitotos y Occidente. Inversa, 1(2), 29-79. Recuperado de: http://es.scribd.com/ doc/40044298/La-Inconmensurabilidad-Conceptual

Vélez-Torres, I. (2013). Desplazamiento y etnicidad: fracasos del multiculturalismo en Colombia. Desacatos, 41: 155-173.

Wachinger, G.; Renn, O.; Begg, C.; Kuhlicke, C. (2013). The Risk Perception ParadoxImplications for Governance and Communication of Natural Hazards. Risk Analysis, 33(6),1049-1065.

Wilches-Chaux, G. (1993). La vulnerabilidad global. En: Maskrey Andrew (Comp.). Los Desastres no son Naturales. Colombia: Tercer Mundo. Recuperado de: http://www. desenredando.org

Wilches-Chaux, G. (1995). Tierra de la gente. Principios orientadores de la Corporación Nasa Kiwe. Revista Desastres y Sociedad, 4, 33-45. Recuperado de: http://www. desenredando.org/public/revistas/dys/

Wilches-Chaux, G. (2000). En el borde del Caos. Bogotá: Instituto Pensar, CEJA Centro Editorial Javeriano.

Zeiderman, A. y Ramírez, L. (2010). Apocalipsis anunciado: un viraje en la política de riesgo en Colombia a partir de 1985. Revista de Ingeniería, 31, 119-131. 
Recepción: 16 de abril de 2015

Evaluación: 4 de julio de 2015

Aprobación: 18 de agosto de 2015 